\title{
Report of A Post-Fracture Conversion Case of: Lone Bone Cyst to Aneurysmal Bone Cyst
}

\section{Raymundo G González Quintanilla*}

Orthopedics' and Traumatology, Mexico

*Corresponding Author: Raymundo G González Quintanilla, Orthopedics' and Traumatology, Mexico.

Received: November 15, 2019; Published: November 19, 2019

DOI: 10.31080/ASOR.2019.02.0123

\section{Concept}

The most common benign cavitation lesion epiphysiary, with no apparent cause of prenominal in bones in growth; It was described in 1904 by Von Mikulicz [1].

Location

In adolescence metaphysical long bones are reported $1 . \mathrm{Hu}-$ merus, 2. Femur, 3. Tibia. [2], then it is diaphysiary as it continues to move away from the epiphysis's.

\section{Frequency}

Relatively commonly in institutional practice, most common in hospitals of concentration. Very variable size in some varieties are called Q.O.S. giants.

Content

It is a liquid with the same chemical components of the blood, without ogical cytol; They compare it to the finding of membrane limiting the pathologists and itis due to a hemodynamic conflict of anterior-venous blood, as exudate with occasional trabecula's is of previous residues in the lesion.

Clinic

Usu mathematic or occasionally increasing regional volume acom pathological fracture diaper in some cases or radiological finding. Auscultation- blow if it is very rare superficial in its frequency.

\section{Evolution}

It usually respects the epiphysis and tends to grow towards diaphysis, it is called 1 . active to the placed in the metaphysis and 2.passive to the diaphasia; F pathological fracture of accrued with its size and located or tends to disappear in some cases? Re-rtan
4 cases of sarcomatous degeneration in the literary area very questionable in my opinion [2].

Diagnosis by complementary studios; Rays of the fluticasone, without periostea reaction. Sign of fragment fell into fractures. I.R.M. in the study are levels, it is difficult to keep without moving the child and deserves in some sedation.

\section{Treatments}

Alternatives 1. Surveillance, 2. Perforations, 3. Injections of material such as steroids, there are some reports of repeating it up to 4 times, 4. Fiatologic Fracture P if accompanied. 5. Injections of autologous bone moil, 6 . Some have used cryosurgery, 7 Used before bench bone or autologous in some cases.

\section{Evolution}

Results in the literature are reported, when there is no fracture that's injections with steroids, offer better successes proportionally in\% practicing the 2 methods of a.- bone medulla and 2.- steroids $[5]$.

In his teaching and in some years as head of art education of the musculoskeletal service from 1968 to 1994 at the University Hospital of the U.A.N.L. School of Medicine. of Monterrey Nuevo Leon Mexico, the number of patients with Quiste Oseo Simple was very numerous and it was as well as possibly starting how to solve such cases; Drugs for our sparse economy sunbathed another, less expensive alternative. In 2001 i presented him at our national congress having as a guest Dr. H. Mankind and we present our case of 28 cases in 22 patients and was obtained in + - 70\% of patients; EL I consider that if the steroid injection was sufficient in the reports of the literature, to get healing then there was no case of trying to 
mean medulla; However studies that I had done previous showed that the material content of medium withinjectedtraste is absorbed be in24 hours, showndothis with consecutive x-rays after emptying the contents and then injecting the bone mist autologin the same patient with the same amount extracted.

I now report this conversion case into private practice in those years. It was accompanied by pathological fracture and vigile until it healed and 8 months later the conversion happened.

\section{Patient}

Male of 13 1/2 years old, healthy, that when he was playing defeats, enduring his right hand and elbow flexed his upper limb, felt a thunder with pain in the shoulder with the effort and when assessing it was the wet lyrical physiary lesion; Fue treated conservatively with an immobilizer until its complete healing.

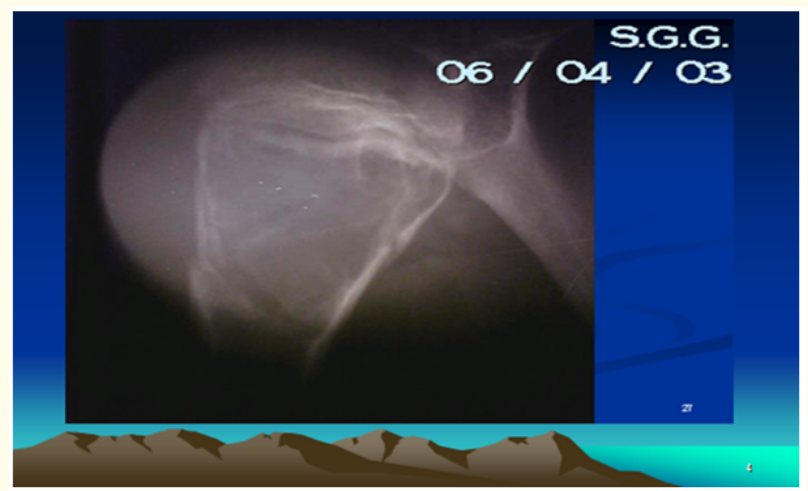

Figure 1: Patient: When playing overdue in the right shoulder, he felt a thunder with pain, was immobilized with a ferula.

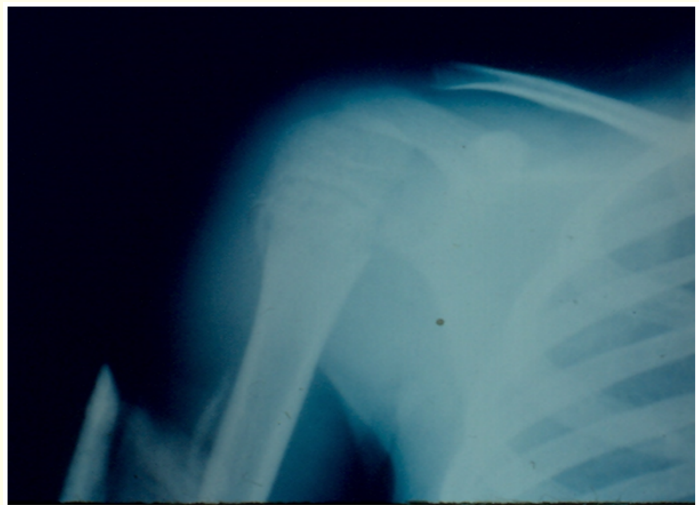

Figure 2: The periostic reaction is from the healing of the fracture. You can think of OS, because of Codman's triangle, but the story is helpful [4].

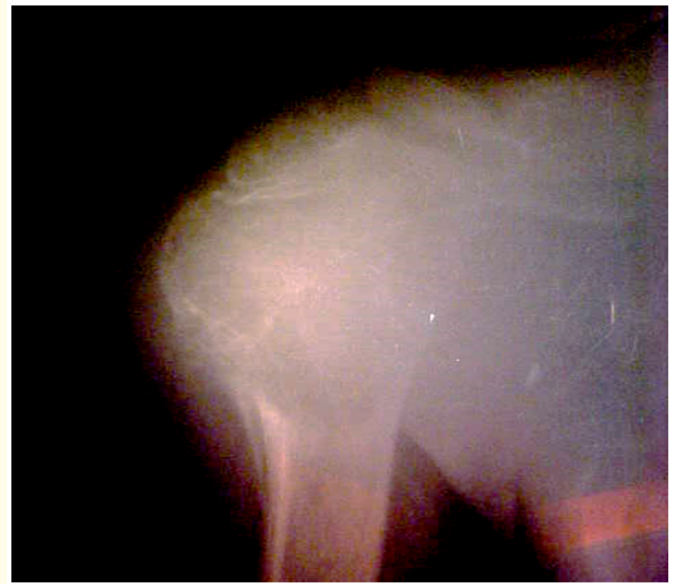

Figure 3: 8 months: Post. - Pathological fracture inica subito growth, in the right shoulder region.
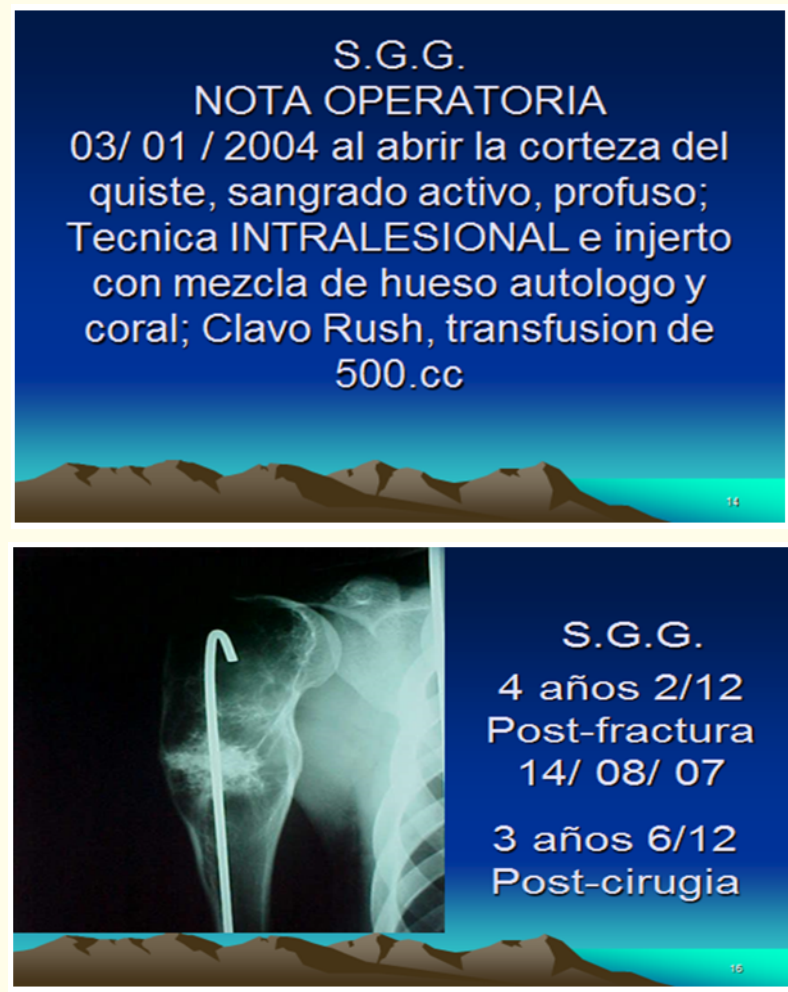

Figure 4: It is fixed with Rush nail to maintain axes and fill the injury space with autologous and coral graft. 


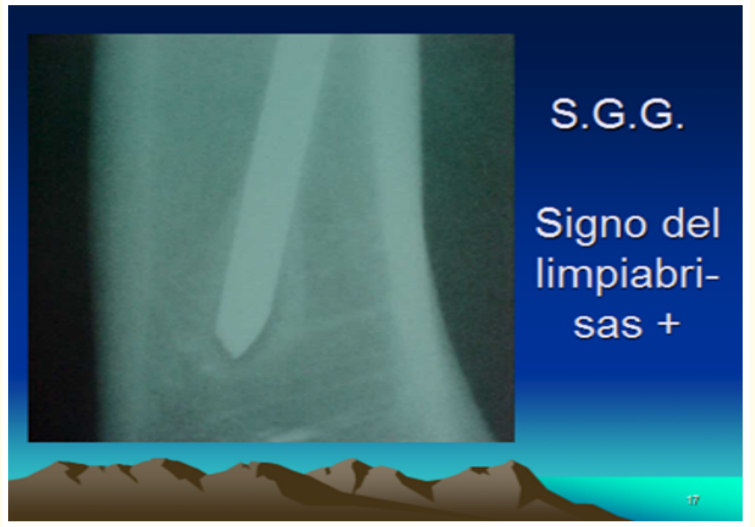

Figure 5: In post-x-ray. - op. the sign of clean breezes is displayed distally in the humerus.

Some Varieties
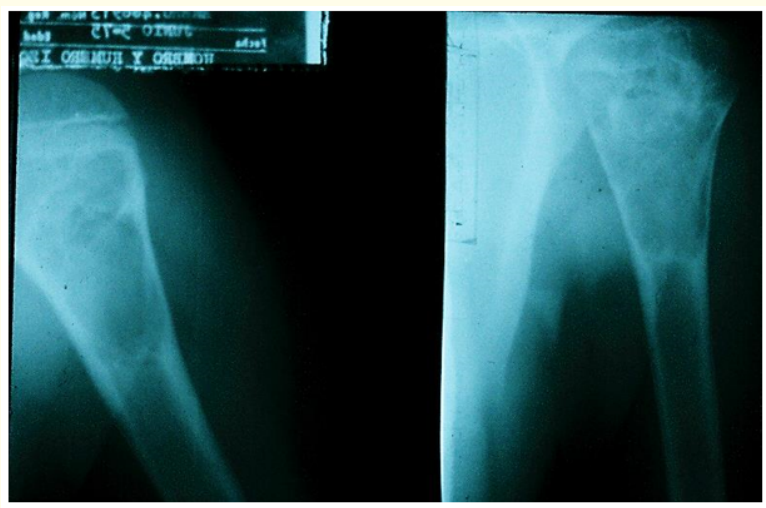

Figure 6: Typical in x-rays: metaphysiary, lithic, some remaining trabecula.

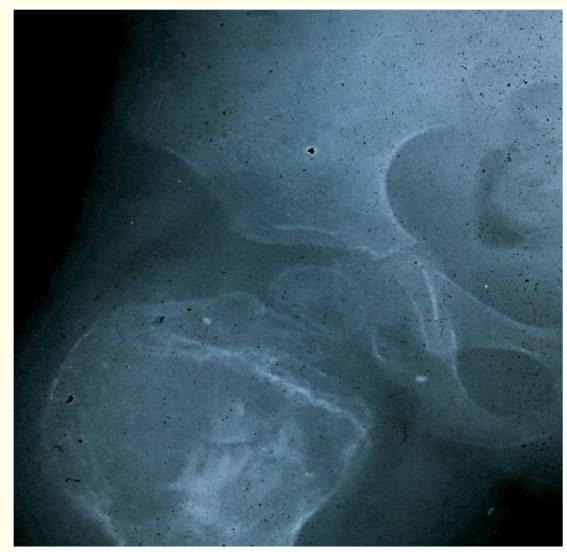

Figure 7: Q.0.S. Giant.

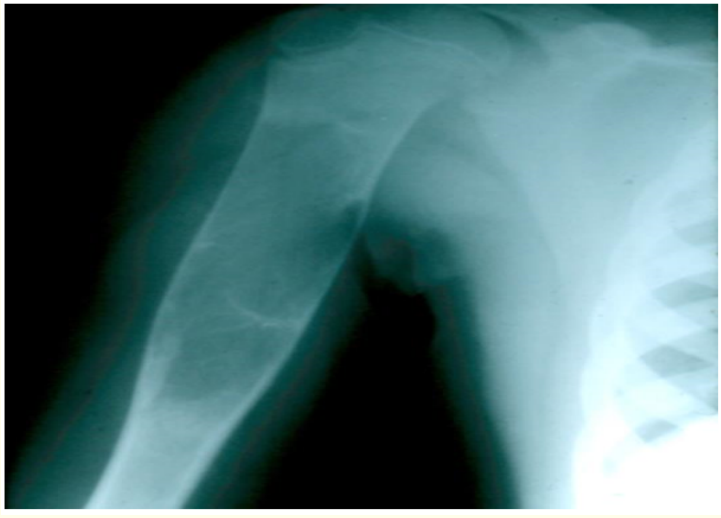

Figure 8: Q.O.S. passive growing becomes a diaphysiary.

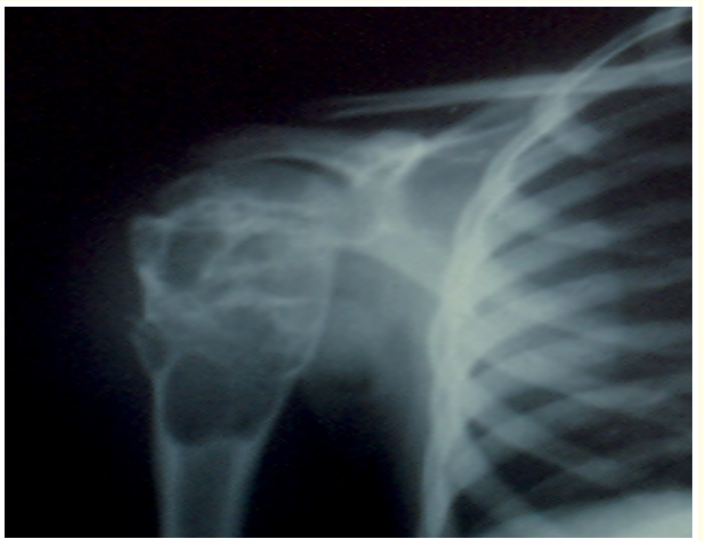

Figure 9: If intralesional trabecula is displayed in Q.0.S., active is probably what remains due to its Litic character.

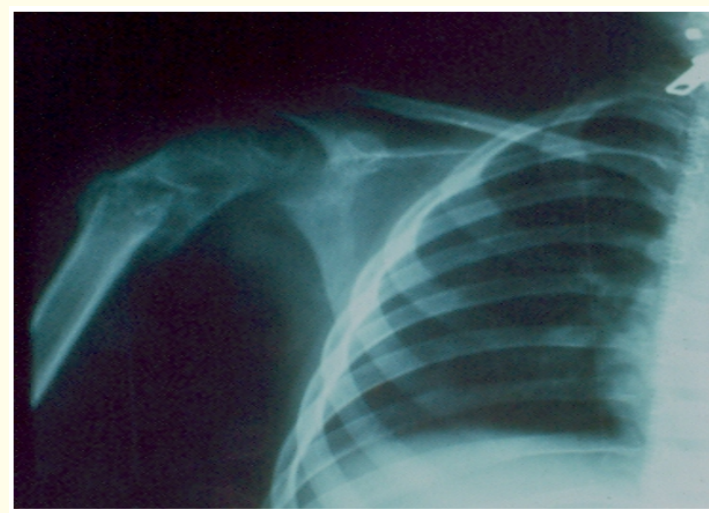

Figure 10: Q.O.S. according to its character of being litic, by its size and location, some are accompanied by pathological fracture [6]. 


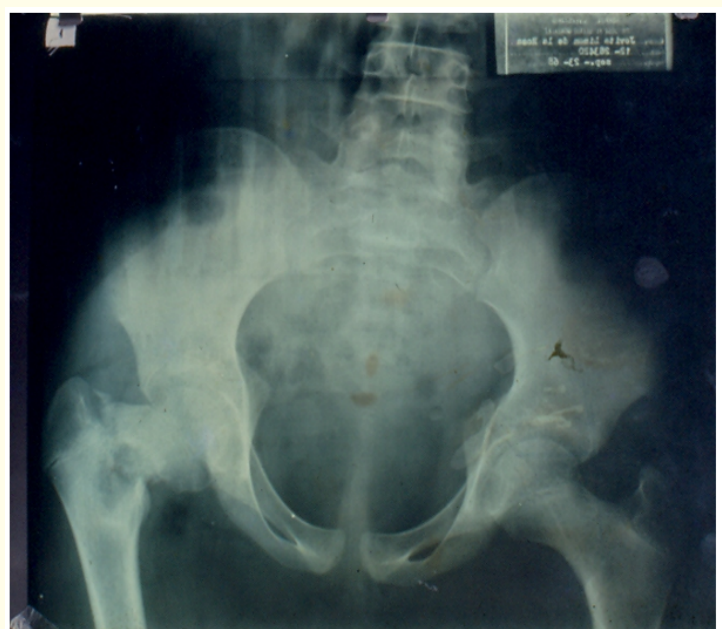

Figure 11: Pathological fracture in the femur that according to the location, in lower limb that is supportive we should analyze the treatment alternatives.

Technically used

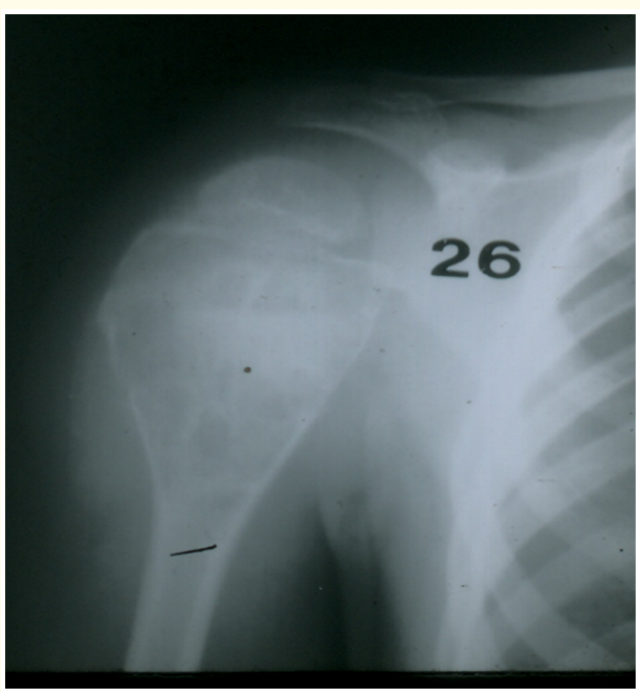

Figure 12: With the same amount of liquid extracted, from the proximal end of the lesion, then it is obtained from the region of the sacral-iliac joint ass; The injected contrast medium (Hipaque) is absorbed + - at 24 hours, keeping the patient seated.

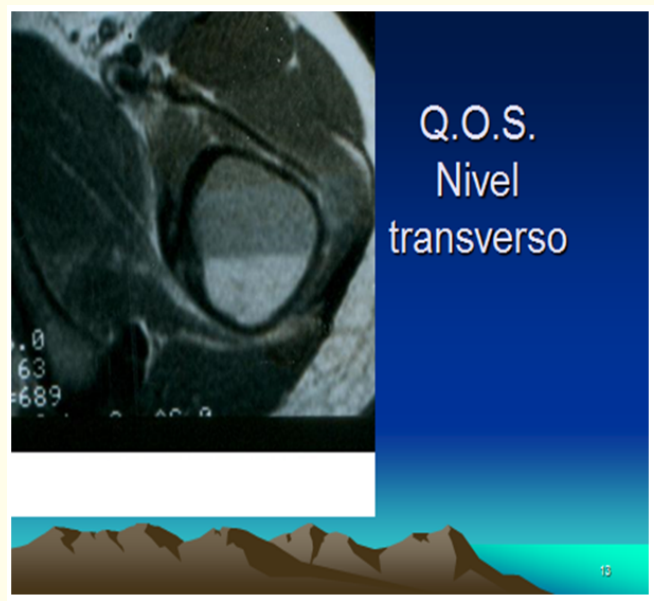

Figure 13: If the content is liquid in I.R.M. of the lesion, thetransverse level confirming the diagnosis is displayed,but it may confuse us with Osteosarcoma telengiectasico [4].

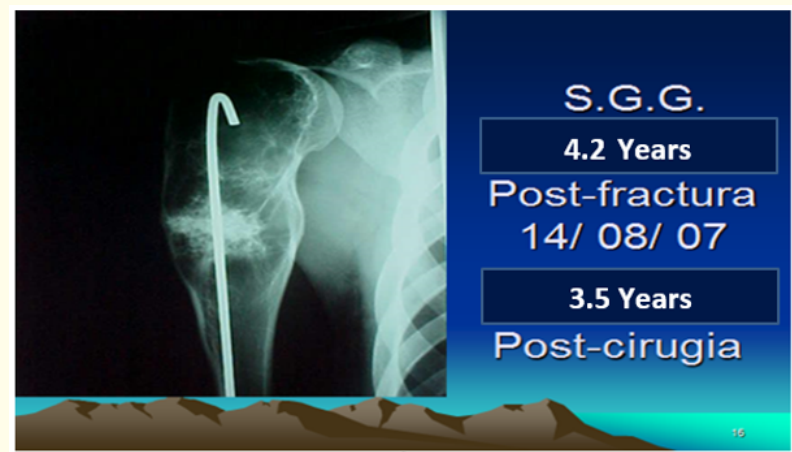

Figure 14: Considering that the injury is benign, it is fixed with a nail to maintain axes of the limb.

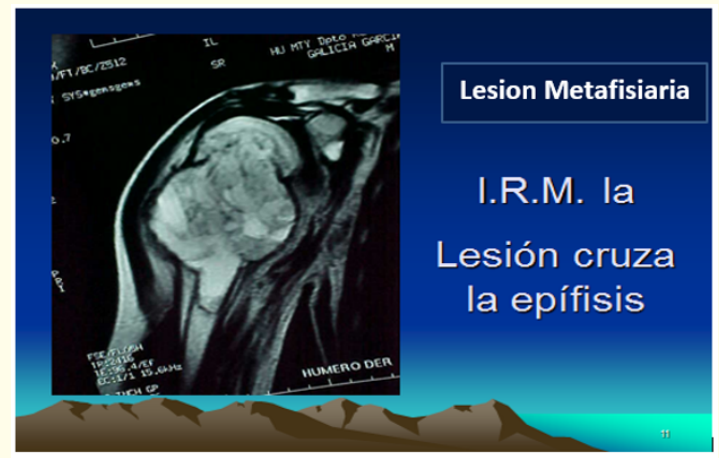

Figure 15: I.R.M. study 8 months post. Scarring. 


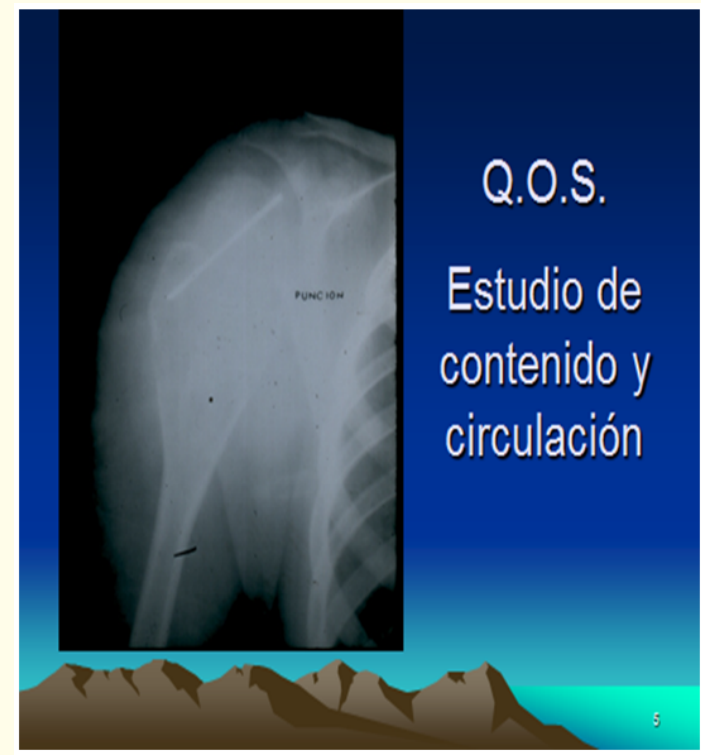

Figure 16: The removal of the fluid from the lesion should always be proximal, it measures how many c.c. and then the bear mdula is injected with the same amount [5].

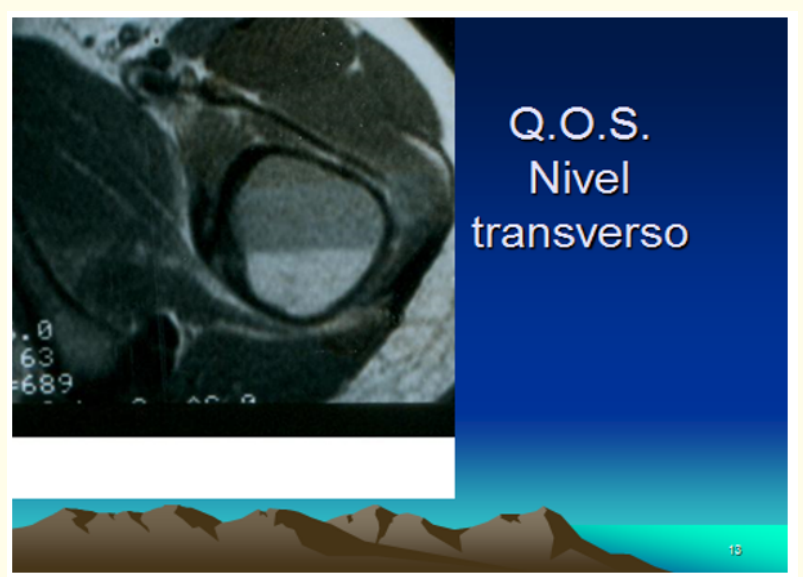

Figure 17: In the I.R.M. study the transverse liquid level confirms the conhad; Osteosarcoma telengiectasico can confuse us [4].

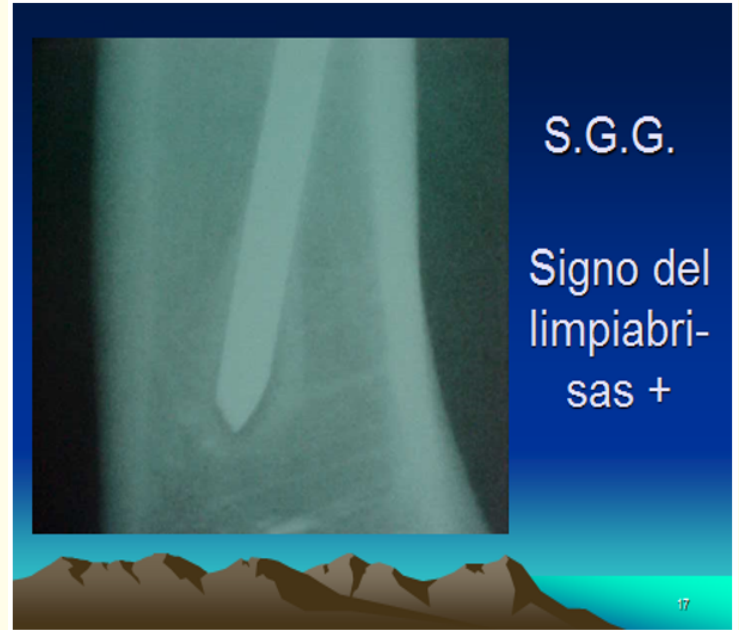

Figure 18: Of adequate length to hold the fragments, this projection is displayed around from the distal end the known: wiper sign.

\section{Conclusion}

1. Report of a patient of pseudo-tumoral injury conversion from Q.O.S. to Q.O.A. very excepcional in his post-fracture evolucion. In addition, it was found metaphysical location towards the epiphysis in another case.

2. Treatment of pathological fracture or finding of the solitary bone cyst, should always be conservador vigilandolo or quirugico according to its location or complication.

3. Ideally the fixation can be temporary, according to age if there is pathological fracture in order to preserve axes of the affected bone.

4. Now in some cases ostoinducers are preferred, avoiding the use of bone.

5. Remember the possibility of the imminent pathological fracture rate, according to the location and size on the Mirels scale $[3,4,6]$.

6. The technique of injecting cortisone could be studied, because according to my experience resolves thethertheus- 
venous conflict?, but how long does it take to absorsee? Pow-

erless mark the cortisone with another means that specifies how long it takes for thatinjury.

7. If defined as Q.O.A. pre.-op. indicates the possibility of embolizing the nutricia artery of the lesion.

\section{Bibliography}

1. Internet.

2. Texto de Dahlin, s Bone Tumors 6 edition Simple cyst (2010): 349.

3. Texto by Mercer Rang Children, s Fractures seconedition (1983): 58.

4. Texto de Dalinh, s bone tumors, 6 edition (2010): 141.

5. Journal of Bone and Joint Surgery U.S.A., (2008): 722.

6. Rockwood and Green, 5 edition, 1 volumen 565.

Volume 2 Issue 12 December 2019

(C) All rights are reserved by Raymundo G González Quintanilla.

Citation: Raymundo G González Quintanilla. "Report of A Post-Fracture Conversion Case of: Lone Bone Cyst to Aneurysmal Bone Cyst". Acta Scientific Orthopaedics 2.12 (2019): 08-13. 